\title{
Spin Period Variations of the White Dwarf in FO Aqr/H2215-086
}

\author{
Thomas Augusteijn \\ European Southern Observatory \\ Casilla 19001, Santiago 19, Chile
}

ABSTRACT. An investigation of the rotation period variation of the white dwarf in FO Aqr/ H2215-086 is presented. A cubic ephemeris is derived for the arrival times of maxima of the pulse light curve which is associated with the white dwarf rotation. Potential problems, resulting from variations of the pulse arrival time as function of orbital phase, and from the change with wavelength of the pulse light curve shape, are investigated. A possible explanation is given for the second effect, being caused by a variable contribution of a modulation at the beat period (of the pulse and orbital periods) as a function of orbital phase.

The magnetic systems form a subclass of the cataclysmic variables. In DQ Her type systems the accretion stream on to the white dwarf is dominated by the magnetic field of the white dwarf. These systems differ from AM Her type systems in that the magnetic interaction with the companion is not sufficiently strong to bring about synchronization of the white dwarf with the orbital period. In DQ Her systems $P_{\text {spin }} \ll P_{\text {orb }}$.

The role of magnetic white dwarfs in the nova phenomenon has recently received increased attention because nova V1500 Cyg was found to be an AM Her type system [1]. Also Williams [2] recently argues that the presence of a significant magnetic field may be an important parameter.

Four of the dozen DQ Her systems, GK Per, V603 Aql, DQ Her and V533 Her are known classical novae. All the other magnetic systems are classified as nova-like. Changes in $P_{\text {spin }}$ have been observed in six DQ Her systems. The most plausible explanation for these changes are variations in the mass transfer rate $\dot{M}$. The period changes indicate time scales of changes in $\dot{M}$ of less than $10^{5}-10^{6} \mathrm{yr}$ [3]. These changes are of special interest in relation to the hibernation model of Shara et al. (e.g. [4]) for cataclysmic variables. As the accretion torques on the white dwarf is exerted via the interaction of the accreting matter with the magnetic field, the period changes also give a (model dependent) estimate of the magnetic field strength.

FO Aqr is a classical example of a DQ Her type system. The optical light curve shows a clear 20.9 min pulsation (also seen in X-rays), identified with the rotation period of the white dwarf, and a slower modulation with the $4.85 \mathrm{hr}$ orbital period. A change in the rotation period period was first determined by Pakull and Beuermann [5], who also detected a difference in the shape of the pulse light curve on either side of the Balmer jump. Recently Osborne and Mukai [6] obtained a significant value for $\ddot{P}$.

To look more closely at the shape of the pulse light curve and the period changes, an investigation was started using simultaneous five colour (VBLUW) photometry with effective wavelengths ranging from $5467 \AA$ to $3255 \AA$. The data were obtained in ' 83 , ' 84 , ' 85 , ' 86 , and ' 88 , and totaled $\approx 10000$ integrations of $16 \mathrm{~s}$ in each of the five pass-bands. The ' 84 data set alone consists of $\approx 4300$ points obtained over a 33 day period.

The arrival time of maximum light with respect to a linear fit is shown in Fig. 1. Crosses indicate values obtained for each of the five years of VBLUW observations, and squares indicate values taken from the literature. From a cubic fit of these arrival times, spanning in total $7.4 \mathrm{yr}$, the following ephemeris is derived:

$$
\begin{aligned}
T_{\max }(\mathrm{HJD})= & 2446256.982635(91)+0.0145191487(29) N+ \\
& +0.72(29) \cdot 10^{-13} N^{2}-4.07(45) \cdot 10^{-18} N^{3}
\end{aligned}
$$


Both the quadratic and cubic terms are significantly different from the values given by Osborne and Mukai, the quadratic term being smaller and the cubic term being bigger. The value of both these terms are mainly determined by the last three points. The relatively large uncertainties in these points, together with a somewhat different approach used here, can probably for a large part account for the difference of the quadratic and cubic terms, as compared to those of Osborne and Mukai.

However, a problem with the determination of arrival times could be the above mentioned difference in the shape of the light curve on either side of the Balmer jump and, as noted by Osborne and Mukai, the variation in arrival time of the pulse as function of orbital phase. As the values in the literature were determined from data obtained with a variety of photometric systems (UBV(RI), white light, and VBLUW), these potential problems were studied using the '84 VBLUW data set. This data set is especially suitable being sufficiently large, taken in one photometric system, and obtained in a short period with respect to variations in the pulse period.

In Fig. 2 the average normalized pulse light curves of the '84 data set are shown. They are ordered from $W$ down with increasing effective wavelength, the $L$ band being on the Balmer jump. In can clearly be seen that the shape of the light curve changes. Sinusoidal fits to the light curves give a difference in arrival times of the maxima in $\mathrm{V}$ and $\mathrm{B}$ with respect to $\mathrm{U}$ and $\mathrm{W}$ of $0.00062(25) \mathrm{d}$. However, the difference in shape of the light curves on either side of the Balmer jump shows large variations with orbital phase, being not noticeable around phase $\approx 0.3$ and $\approx 0.75$.

The pulse light curves also show variations with the orbital period. In Fig. 3 the amplitude of the light curve in $\mathrm{V}$ as function of the orbital phase is shown. The variations in amplitude are similar in all five colours, and follow closely the orbital light variations. The shape and maxima of the pulse light curves also change with orbital phase and are consistent with an additional modulation with the beat period of the pulse and orbital period. This modulation is in phase with the main pulse around phase $\approx 0.75$, pointing to the hot spot as the reprocessing side of the main pulse whose changing aspect with orbital phase gives rise to the beat modulation. There are also some indications for a second maximum in the beat modulation being in phase with the main pulse around $\approx 0.25$.

It is quite interesting that the orbital phases at which the two possible maxima of the beat modulation are in phase with the main pulse, coincide with the phase at which the difference of the shape in the light curve on either side of the Balmer jump vanishes. Taking into account that the strength of the main pulse changes with orbital phase (see Fig. 3), which probably also is the case for the beat modulation, and considering that the beat modulation has most likely a different spectral distribution from that of the main pulse, this would give a natural explanation for the difference in shape of the pulse light curve on either side of the Balmer jump. This difference would then be the result of the changing of the relative strength of the two modulations with orbital phase. If the relative strength would be constant no effect of the beat modulation on the average main pulse light curve would be seen.

A further study into the behavior of the beat modulation, its cause(s), and the influence of the different variations discussed above on the determination of the arrival times of the pulse light curve maxima is underway.

\section{References:}

[1] Stockman, H.S., Schmidt, G.D., and Lamb, D.Q. 1988. Astrophys. J., 332, 282.

[2] Williams, R.E. 1989. Astr. J., 97, 1752.

[3] Van Amerongen, S., Augusteijn, T., and Van Paradijs, J. 1987. Mon. Not. R. astr. Soc., 228, 377.

[4] Livio, M., and Shara, M.M. 1987. Astrophys. J., 319, 819.

[5] Pakull, M.W., and Beuermann, K. 1987. Astrophys. Space Sci., 130, 641.

[6] Osborne, J.P., and Mukai, K. 1989. Mon. Not. R. astr. Soc., 238, 1233. 


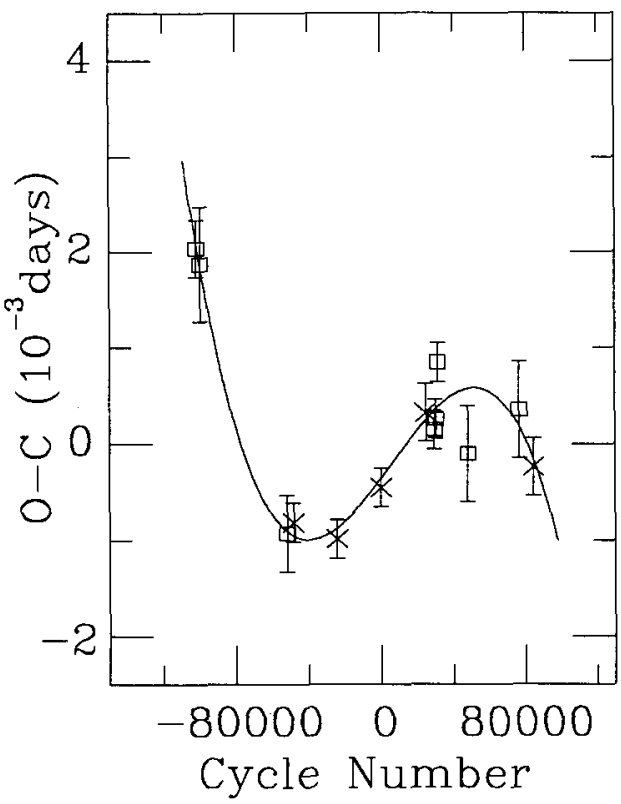

Fig. 1. The arrival times of the pulse with respect to a linear fit. The line is the cubic fit to the data (see text).

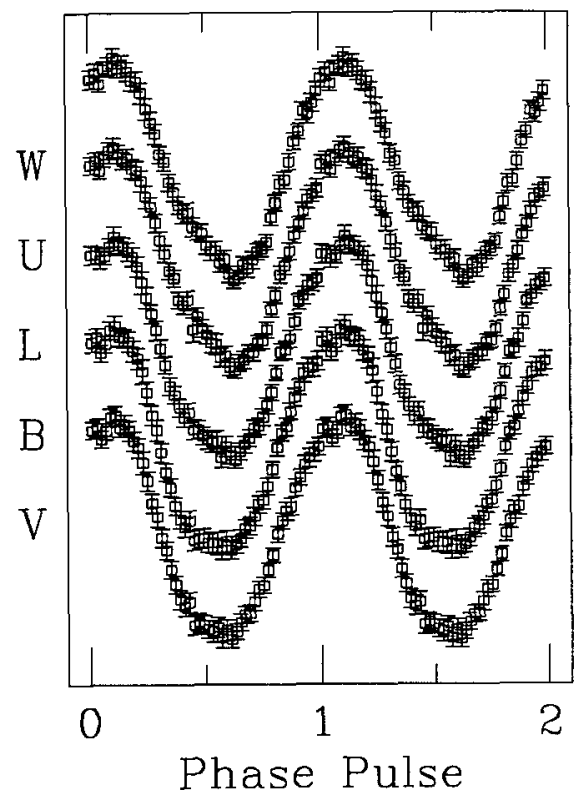

Fig. 2. The normalized average pulse light curves in VBLUW for the '84 data. Notice the change in shape around the minimum in the different pass bands.

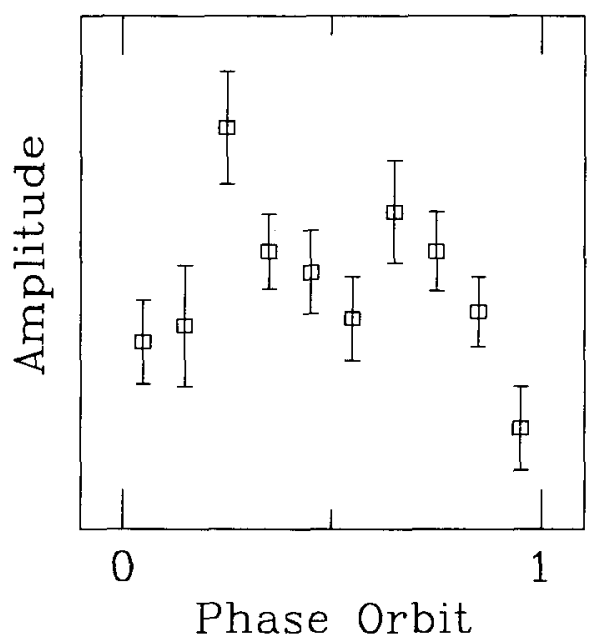

Fig. 3. The amplitude of the main pulse as function of the orbital phase. The variation is very similar in all five pass bands, and follows closely the orbital light variations. 